\title{
Selection Response and Genetic Variability for Yield and its Component Traits of Rice (Oryza sativa L.)
}

\author{
A.K. Choudhary ${ }^{1^{*}}$, Z.A. Haider ${ }^{2}$, K. Prasad $^{2}$, S.B. Mishra ${ }^{3}$, \\ M. Chakraborty ${ }^{2}$ and Niraj Kumar ${ }^{2}$
}

${ }^{1}$ Bhola Paswan Shastri Agricultural College, Department of Plant Breeding and Genetics, Purnea City, Purnea-854 302, India

${ }^{2}$ Birsa Agricultural University, Kanke, Ranchi, Jharkhand, India

${ }^{3}$ Department of Plant Breeding and Genetics, T.C.A. Dholi, Muzaffarpur - 843 121, Bihar, India

*Corresponding author

\section{A B S T R A C T}

\section{Keywords \\ Selection response, Standardized selection, differential and realized heritability \\ Article Info \\ Accepted: \\ 07 March 2018 \\ Available Online: \\ 10 April 2018}

Cross - I exhibited high $\mathrm{h}^{2}$ coupled with moderate genetic advance as per cent of mean for Tw in all selection methods as well as different methods and date of sowing except in HY selection method on $I^{\text {st }}$ date TP followed by LY selection methods on $\mathrm{I}^{\text {st }}$ date DS; and MTL selection methods on $2^{\text {nd }}$ date TP indicating the preponderance of additive gene action as well as non-additive gene action. Cross - II and Cross - III were obtained highest average selection response for all the studied traits in HY followed by LY selection method. Among different methods of selection HY followed by LY selection method may be effective in early segregating generation for Cross - II as well as Cross - III whereas for Cross - I; LY followed by HY selection method may be more effective. It might be probable that delaying selection reduces the frequency of high yielding genotypes resulting in a greater frequency of low yielding genotypes.

\section{Introduction}

Rice (Oryza sativa L.) is the staple food for around 2.5 billion world's population which is about $60 \%$ of the total population and may escalate to 4.6 billion by the year 2050; especially for most of the people of SouthEast Asia. Among the rice growing countries in the world, India has the largest area under rice crop and ranks second in production next to China. It plays vital role in the national food grain supply. It accounts for about $43 \%$ of food grain production in the country. Rice is grown in three seasons in India, where Kharif season accounts for $88 \%$ production while Rabi contributes only $12 \%$ production. Rice is the single most important food crop in India and occupies $44.6 \mathrm{~m}$ ha under rice cultivation Meera et al., (2014). The population growth in most of the Asian countries, except China, continues to be around 2 percent per year. Hence it is very pertinent to critically consider whether the rice production can be further increased to keep pace with population growth. With the current green revolution 
technologies it is estimated that by 2020 at least 115-120 million tons of milled rice is to be produced in India to maintain the present level of self-sufficiency. The total production could be enhanced either by making horizontal expansion in area, which is not possible owing to high population growth, so none of the option left other than vertical expansion, which could be done opting a suitable breeding method.

The early generation testing is one of the best option to reduce the amount of material to be handled in the segregating generations and at the same time retain the good recombinant lines for the traits under improvement. It is also enhanced by selection response which maximises either by selecting the best genotype available in the population or by increasing the rigour of selection. A very rigorous selection may not be desirable as it can eliminate some promising genotypes. Whan et al., (1982) suggested that selection for grain yield in early generation need to be done at many sites simultaneously at an early growth stage. Grain yield is a complex character and is the result of interaction of many variables due to different gene association that might exist in different population and might result in quite different relationships. It is also largely influenced by environment. Further genotype and environmental interaction reduces the effectiveness of early generation selection (Whan et al., (1981). Large environmental differences may lead to failure of parental yield to be indicative of the yield of progeny. The present investigation is aimed to collect the information on effectiveness of the selection in the improvement programme based on the performance of individual groups of three crosses selection from $\mathrm{F}_{2}$ generation, keeping yield/plant, no. of grain /panicle, thousand grain weight, plant height, and panicle/plant with the following objectives. Validation of early generation selection
Assessing effective method of early generation selection.

\section{Materials and Methods}

The experimental material consisted of three crosses viz; BG102/BPT5204, BG102/Naveen and BG102/ Reshmi $\left(\mathrm{F}_{1}\right)$ Commercial Pvt. hybrid (Victory seeds) of rice (Oryza sativa L.). Each of the crosses represented a separate experiment which were obtained from Rice Project, Department of Plant Breeding and Genetics, Birsa Agricultural University, Ranchi, Jharkhand. All the materials come under single cross, except, BG102/ Reshmi $\left(\mathrm{F}_{1}\right)$ Commercial Pvt. hybrid (Victory seeds) which is a three way cross. The $\mathrm{F}_{2}$ materials were grown in nursery on $6^{\text {th }}$ June, 2013. Thirty (30) days old seedlings were transplanted in the puddled field. Out of total $5000 \mathrm{~F}_{2}$ established population, $1000 \mathrm{~F}_{2}$ were tagged randomly after flowering of each cross viz; BG102/BPT5204, BG102/Naveen and BG102/ Reshmi $\left(\mathrm{F}_{1}\right)$ Commercial Pvt. Hybrid (Victory seeds) respectively and represented as Cross- I, Cross- II and Cross- III. The data recording and harvesting of each plants were done separately so that these $F_{2}$ plants be categorized and selected. On the basis of these data 50 plants were selected in each groups viz., high yielder (HY), low yielder (LY), multi trait limit (MTL) and random plant selection (RPS). Each group comprising 50 plants but in MTL having only 48, 49 and 42 plants in each cross viz; Cross- I, Cross- II and Cross- III respectively. High and low yielder plants were selected on the basis of their high and low yield potential however, in MTL group optimum plants were selected by fixing certain traits range viz; PH $(70-110 \mathrm{~cm})$, PPP (5-25), PL (18-35 cm), GPP (80-250) and test wt. (100 seed, 1.9-3.0 g) but in RPS group plant was selected on random basis. These $\mathrm{F}_{2}$ selected plants were grown during kharif, 2014 in RBD with two replication and two 
methods of sowing, such as, direct seeded and transplanted at twenty days interval; each plot measuring $2.7 \times 0.4$ meter size. The row to row distance was kept at $20 \mathrm{~cm}$ while plant to plant distance was maintained at $15 \mathrm{~cm}$. A fertilizer dose of 80: 40: $30 \mathrm{~N}: \mathrm{P}: \mathrm{K} \mathrm{Kg}$ / ha was applied in two parts $40 \mathrm{~kg}$ of $\mathrm{N}$, all phosphate and potash were applied as a basal and the remaining $40 \mathrm{~kg} \mathrm{~N}$ was applied as top dressing in two split doses. The analysis of variance was carried out separately for each trait as per formula suggested by Panse and Sukhatme (1967), phenotypic and genotypic coefficient of variation by Burton (1952), heritability (Broad sense) and genetic advance as per cent of mean were estimated by the formula as suggested by Johanson et al., (1955). Standardized selection differential, response to selection and realized heritability were estimated as per Falconer (1989).

\section{Results and Discussion}

The results obtained with respect to genetic variability of different traits of $\mathrm{F}_{3}$ generations are given in the (Table 1, 2 and 3). Knowledge on nature and magnitude of genotypic and phenotypic variability present in any crop species plays an important role in formulating successful breeding programmes (Allard, 1960). Sivasubramanian and Menon (1973) also highlighted the importance of variability in early segregating generations and suggested that magnitude of genotypic coefficient of variability and phenotypic coefficient of variability should be given importance. Jennings et al., (1979) suggested that crosses which will realise early homozygosity are ideally suited for further breeding work.

In general PCV was bit higher than GCV in all crosses which indicates additive effect of environment on the expression of the trait. Similar finding have been reported earlier by Mohan and Chauhan (2011), Praveen et al., (2010), Chakraborty and Chakraborty (2010) and Gala et al., (2016).

In $50 \mathrm{~F}_{3}$ progenies of Cross - I, high $\mathrm{h}^{2}$ coupled with moderate genetic advance as per cent of mean has been recorded for Tw in all selection methods as well as different methods and date of sowing except in HY group on $\mathrm{I}^{\text {st }}$ date TP followed by LY selection methods on $\mathrm{I}^{\text {st }}$ date DS; and MTL selection methods on $2^{\text {nd }}$ date $\mathrm{TP}$, indicating the preponderance of additive gene action as well as non-additive gene action. For trait GPP recorded high $\mathrm{h}^{2}$ coupled with high GA as per cent of mean in $\mathrm{I}^{\text {st }}$ and $2^{\text {nd }}$ date of DS as well as TP respectively under HY selection methods whereas high GA along with moderate $h^{2}$ was obtained in both date of sowing under same selection method indicating the preponderance of additive gene action. Similar finding earlier have been reported by Singh et al., (2013), Dutta et al., (2013), Rajendra et al., (2013), Tuhina et al., (2015) and Lingaiah (2015), whereas for most of characters have been recorded high $h^{2}$ coupled with low to moderate genetic advance as per cent of mean in HY and LY selection methods which is might be due to preponderance of additive and non- additive gene action. Similar finding earlier has been reported by Chakraborty and Chakraborty (2010) and Sanghera et al., (2013).

In Cross - II of $50 \mathrm{~F}_{3}$ progenies high $\mathrm{h}^{2}$ coupled with high GA as per cent of mean has been recorded for Tw in LY group of $\mathrm{I}^{\text {st }}$ date TP and $2^{\text {nd }}$ date TP along with MTL and RPS.GYP plant had also exhibited high $h^{2}$ coupled with high genetic advance as per cent of mean in all most all the selection methods except in HY group and $\mathrm{I}^{\text {st }}$ date DS and TP whereas in Cross-I II; for traits viz; DFF, PH, PPP, GPP, GYP plant and GYP plot were exhibited high $\mathrm{h}^{2}$ coupled with high GA as per cent of mean in MTL and RPS selection methods as well as both dates of Sivasubramanian and Menon (1973) planting. 
Table.1 Genetic parameters of $50 \mathrm{~F}_{3}$ progenies selected from $\mathrm{F}_{2}$ population Cross- I (BG102/BPT5204) based on different selection indices and grown under different crop ecology

\begin{tabular}{|c|c|c|c|c|c|c|c|c|c|c|c|c|c|c|c|c|c|}
\hline \multirow[t]{3}{*}{ Characters } & \multirow{3}{*}{$\begin{array}{c}\text { Genetic } \\
\text { parameters }\end{array}$} & \multicolumn{4}{|c|}{$\mathrm{I}^{\text {st }}$ method of selection (HY) } & \multicolumn{4}{|c|}{$2^{\text {nd }}$ method of selection (LY) } & \multicolumn{4}{|c|}{$3^{\text {rd }}$ method of selection (MTL) } & \multicolumn{4}{|c|}{$4^{\text {th }}$ method of selection (RPS) } \\
\hline & & \multicolumn{2}{|c|}{$I^{\text {st }}$ Date } & \multicolumn{2}{|c|}{$2^{\text {nd }}$ Date } & \multicolumn{2}{|c|}{$\mathbf{I}^{\text {st }}$ Date } & \multicolumn{2}{|c|}{$2^{\text {nd }}$ Date } & \multicolumn{2}{|c|}{$I^{\text {st }}$ Date } & \multicolumn{2}{|c|}{$2^{\text {nd }}$ Date } & \multicolumn{2}{|c|}{ I $^{\text {st }}$ Date } & \multicolumn{2}{|c|}{$2^{\text {nd }}$ Date } \\
\hline & & DS & $\mathbf{T P}$ & DS & TP & DS & $\mathbf{T P}$ & DS & TP & DS & TP & DS & $\mathbf{T P}$ & DS & TP & DS & TP \\
\hline \multirow[t]{9}{*}{ DFF } & $\mathbf{h}^{2}(\%)$ & 74.59 & 76.75 & 42.53 & 67.64 & 73.21 & 22.03 & 9.60 & 64.28 & 65.43 & 84.12 & 65.98 & 84.26 & 91.13 & 71.48 & 78.87 & 86.18 \\
\hline & GA $(\%)$ & 12.93 & 7.31 & 5.08 & 5.95 & 6.72 & 2.19 & 1.14 & 4.94 & 5.34 & 6.51 & 5.38 & 6.52 & 8.66 & 7.18 & 7.75 & 8.30 \\
\hline & $\operatorname{GCV}(\%)$ & 4.40 & 4.05 & 3.78 & 3.51 & 3.83 & 2.26 & 1.78 & 2.99 & 3.82 & 3.75 & 3.94 & 3.37 & 5.31 & 4.52 & 5.24 & 4.28 \\
\hline & PCV (\%) & 5.10 & 4.62 & 5.79 & 4.26 & 4.48 & 4.82 & 5.75 & 3.72 & 4.73 & 4.08 & 4.85 & 3.67 & 5.56 & 5.35 & 5.90 & 4.61 \\
\hline & Mean & 72 & 80 & 70 & 89 & 69 & 78 & 68 & 87 & 70 & 80 & 70 & 89 & 70 & 78 & 69 & 88 \\
\hline & Min. & 91 & 97 & 87 & 106 & 83 & 91 & 82 & 101 & 86 & 92 & 82 & 101 & 89 & 96 & 87 & 106 \\
\hline & Max. & 90 & 96 & 87 & 106 & 69 & 77 & 68 & 87 & 85 & 91 & 82 & 101 & 88 & 96 & 86 & 105 \\
\hline & CD at 0.05 & 4 & 4 & 6 & 5 & 4 & 7 & 8 & 4 & 4 & 4 & 3 & 3 & 3 & 5 & 4 & 3 \\
\hline & CV \% & 3 & 2 & 6 & 2 & 2 & 4 & 5 & 2 & 3 & 3 & 2 & 1 & 2 & 3 & 3 & 2 \\
\hline \multirow[t]{9}{*}{ PH (cm) } & $h^{2}(\%)$ & 5.35 & 16.38 & 10.45 & 3.73 & 11.07 & 18.69 & 34.66 & 37.65 & 15.98 & 11.21 & 12.78 & 27.98 & 11.34 & 10.35 & 70.42 & 13.12 \\
\hline & GA (\%) & 0.84 & 2.20 & 1.67 & 0.71 & 1.52 & 3.47 & 5.68 & 6.09 & 2.61 & 1.68 & 2.23 & 5.18 & 2.25 & 2.07 & 14.00 & 2.91 \\
\hline & $\operatorname{GCV}(\%)$ & 1.75 & 2.63 & 2.50 & 1.78 & 2.22 & 3.89 & 4.68 & 4.82 & 3.11 & 2.39 & 2.97 & 4.66 & 3.23 & 3.11 & 8.09 & 3.90 \\
\hline & $\operatorname{PCV}(\%)$ & 7.59 & 6.50 & 7.73 & 9.22 & 6.69 & 9.01 & 7.95 & 7.85 & 7.78 & 7.12 & 8.32 & 8.81 & 9.61 & 9.69 & 9.65 & 10.76 \\
\hline & Mean & 122 & 121 & 122 & 122 & 122 & 121 & 122 & 122 & 115 & 115 & 116 & 115 & 121 & 123 & 124 & 124 \\
\hline & Min. & 108 & 106 & 108 & 104 & 109 & 108 & 105 & 109 & 101 & 103 & 102 & 94 & 101 & 96 & 96 & 99 \\
\hline & Max. & 133 & 132 & 135 & 138 & 137 & 160 & 139 & 137 & 129 & 131 & 134 & 135 & 148 & 139 & 147 & 145 \\
\hline & CD at 0.05 & 22 & 14 & 20 & 22 & 16 & 20 & 16 & 15 & 17 & 18 & 16 & 18 & 24 & 23 & 13 & 25 \\
\hline & CV \% & 9 & 6 & 8 & 9 & 6 & 8 & 6 & 6 & 7 & 8 & 7 & 7 & 10 & 9 & 5 & 10 \\
\hline \multirow[t]{9}{*}{ PPP } & $h^{2}(\%)$ & 9.93 & 23.53 & 25.41 & 27.26 & 21.85 & 41.82 & 15.61 & 38.68 & 27.16 & 50.69 & 19.75 & 27.22 & 43.97 & 11.91 & 53.95 & 14.88 \\
\hline & GA (\%) & 3.78 & 9.56 & 8.69 & 12.00 & 5.64 & 16.73 & 5.42 & 15.24 & 10.09 & 35.25 & 14.98 & 11.59 & 20.28 & 5.84 & 26.70 & 7.15 \\
\hline & GCV (\%) & 5.81 & 10.87 & 8.08 & 9.98 & 7.38 & 12.29 & 6.45 & 12.54 & 10.40 & 24.04 & 15.57 & 11.01 & 13.18 & 8.97 & 16.76 & 8.91 \\
\hline & PCV (\%) & 18.45 & 22.41 & 16.03 & 19.12 & 15.79 & 19.00 & 16.33 & 20.17 & 19.97 & 33.77 & 35.06 & 21.12 & 19.88 & 26.01 & 22.82 & 23.10 \\
\hline & Mean & 12 & 11 & 11 & 12 & 10 & 10 & 10 & 10 & 9 & 9 & 9 & 8 & 10 & 9 & 9 & 9 \\
\hline & Min. & 9 & 8 & 8 & 4 & 7 & 7 & 8 & 7 & 6 & 6 & 7 & 1 & 3 & 6 & 7 & 7 \\
\hline & Max. & 16 & 16 & 15 & 17 & 12 & 14 & 13 & 12 & 13 & 18 & 16 & 12 & 13 & 14 & 15 & 25 \\
\hline & CD at 0.05 & 4 & 4 & 3 & 4 & 3 & 3 & 3 & 3 & 3 & 6 & 4 & 3 & 3 & 4 & 3 & 4 \\
\hline & CV \% & 18 & 20 & 14 & 16 & 14 & 14 & 15 & 16 & 17 & 31 & 24 & 18 & 15 & 24 & 15 & 21 \\
\hline GPP & $\mathbf{h}^{2}(\%)$ & 73.41 & 58.96 & 69.99 & 52.46 & 57.28 & 41.79 & 47.27 & 40.82 & 50.47 & 12.35 & 37.14 & 49.09 & 40.39 & 56.02 & 47.36 & 37.68 \\
\hline
\end{tabular}




\begin{tabular}{|c|c|c|c|c|c|c|c|c|c|c|c|c|c|c|c|c|c|}
\hline & GA (\%) & 39.07 & 25.91 & 30.92 & 21.59 & 24.72 & 17.22 & 20.21 & 15.90 & 24.26 & 6.24 & 15.43 & 20.71 & 18.54 & 22.30 & 23.37 & 16.06 \\
\hline & GCV (\%) & 22.13 & 16.38 & 17.94 & 14.46 & 15.85 & 12.92 & 14.26 & 12.27 & 16.28 & 8.47 & 12.08 & 14.10 & 14.16 & 14.46 & 14.60 & 12.70 \\
\hline & PCV (\%) & 25.83 & 21.33 & 21.44 & 19.97 & 20.94 & 19.99 & 20.75 & 19.20 & 22.91 & 24.12 & 19.82 & 20.12 & 22.28 & 19.32 & 21.22 & 20.68 \\
\hline & Mean & 81 & 103 & 99 & 88 & 88 & 89 & 90 & 89 & 85 & 86 & 88 & 84 & 89 & 83 & 89 & 89 \\
\hline & Min. & 36 & 54 & 56 & 18 & 50 & 66 & 52 & 50 & 54 & 57 & 59 & 25 & 62 & 47 & 64 & 69 \\
\hline & Max. & 113 & 143 & 132 & 118 & 135 & 131 & 138 & 135 & 141 & 124 & 116 & 110 & 135 & 112 & 133 & 176 \\
\hline & CD at 0.05 & 22 & 28 & 24 & 24 & 25 & 27 & 28 & 26 & 28 & 28 & 39 & 24 & 31 & 21 & 27 & 29 \\
\hline & CV \% & 13 & 14 & 12 & 14 & 14 & 15 & 15 & 15 & 16 & 16 & 23 & 14 & 17 & 13 & 15 & 16 \\
\hline \multirow[t]{9}{*}{ TW(g) } & $h^{2}(\%)$ & 76.00 & 84.77 & 82.59 & 96.42 & 95.35 & 92.00 & 93.39 & 89.07 & 86.38 & 92.10 & 88.87 & 95.94 & 62.27 & 86.06 & 79.19 & 86.47 \\
\hline & GA (\%) & 12.20 & 14.56 & 13.13 & 26.33 & 20.97 & 19.94 & 21.24 & 21.17 & 15.98 & 16.76 & 16.17 & 27.90 & 10.01 & 14.42 & 12.86 & 13.07 \\
\hline & $\operatorname{GCV}(\%)$ & 6.79 & 7.67 & 7.01 & 13.01 & 10.42 & 10.09 & 10.66 & 10.83 & 8.17 & 8.30 & 8.15 & 13.55 & 6.15 & 7.54 & 7.01 & 6.82 \\
\hline & PCV (\%) & 7.79 & 8.33 & 7.71 & 13.25 & 10.67 & 10.51 & 11.04 & 11.48 & 8.80 & 8.65 & 8.65 & 13.83 & 7.79 & 8.13 & 7.88 & 7.33 \\
\hline & Mean & 22 & 21 & 22 & 22 & 21 & 21 & 21 & 21 & 21 & 21 & 21 & 21.26 & 22 & 22 & 22 & 21 \\
\hline & Min. & 18 & 18 & 18 & 18 & 17 & 18 & 17 & 18 & 18 & 17 & 18 & 17.89 & 19 & 19 & 19 & 19 \\
\hline & Max. & 25 & 25 & 25 & 26 & 27 & 27 & 27 & 27 & 25 & 25 & 25 & 25.41 & 25 & 26 & 26 & 24 \\
\hline & CD at 0.05 & 2 & 1 & 1 & 1 & 1 & 1 & 1 & 2 & 1 & 1 & 1 & 1.17 & 2 & 1 & 2 & 1 \\
\hline & CV \% & 4 & 3 & 3 & 3 & 2 & 3 & 3 & 4 & 3 & 3 & 2 & 2.79 & 5 & 3 & 4 & 3 \\
\hline \multirow{9}{*}{$\begin{array}{l}\text { GYP Plant } \\
\text { (g) }\end{array}$} & $h^{2}(\%)$ & 46.14 & 28.47 & 35.74 & 50.26 & 50.32 & 59.74 & 32.16 & 25.01 & 49.89 & 53.38 & 31.42 & 17.23 & 31.57 & 29.84 & 41.84 & 16.05 \\
\hline & GA (\%) & 18.45 & 11.58 & 14.92 & 25.29 & 28.25 & 25.77 & 10.73 & 6.38 & 45.55 & 43.06 & 22.30 & 6.26 & 14.67 & 10.76 & 28.40 & 4.60 \\
\hline & $\operatorname{GCV}(\%)$ & 13.18 & 10.53 & 12.10 & 17.31 & 19.32 & 16.18 & 9.18 & 6.44 & 30.80 & 28.14 & 19.02 & 7.19 & 12.67 & 9.55 & 21.30 & 5.56 \\
\hline & PCV (\%) & 19.40 & 19.75 & 20.25 & 24.42 & 27.24 & 20.93 & 16.18 & 12.87 & 43.61 & 38.51 & 33.94 & 17.31 & 22.55 & 17.48 & 32.94 & 13.89 \\
\hline & Mean & 22 & 21 & 22 & 22 & 21 & 21 & 21 & 21 & 21 & 21 & 21 & 21.26 & 22 & 22 & 22 & 21 \\
\hline & Min. & 18 & 18 & 18 & 18 & 17 & 18 & 17 & 18 & 18 & 17 & 18 & 17.89 & 19 & 19 & 19 & 19 \\
\hline & Max. & 25 & 25 & 25 & 26 & 27 & 27 & 27 & 27 & 25 & 25 & 25 & 25.41 & 25 & 26 & 26 & 24 \\
\hline & CD at 0.05 & 2 & 1 & 1 & 1 & 1 & 1 & 1 & 2 & 1 & 1 & 1 & 1.17 & 2 & 1 & 2 & 1 \\
\hline & CV \% & 4 & 3 & 3 & 3 & 2 & 3 & 3 & 4 & 3 & 3 & 2 & 2.79 & 5 & 3 & 4 & 3 \\
\hline \multirow{9}{*}{$\begin{array}{l}\text { GYP Plot } \\
\text { (g) }\end{array}$} & $h^{2}(\%)$ & 44.28 & 35.19 & 57.01 & 34.98 & 19.01 & 18.99 & 49.76 & 19.01 & 14.13 & 13.36 & 16.44 & 33.57 & 4.09 & 12.41 & 21.24 & 14.97 \\
\hline & GA (\%) & 17.23 & 15.01 & 29.53 & 16.42 & 6.59 & 7.01 & 25.07 & 6.59 & 4.12 & 4.17 & 6.61 & 12.64 & 1.37 & 4.01 & 12.50 & 5.65 \\
\hline & $\operatorname{GCV}(\%)$ & 12.57 & 12.27 & 18.98 & 13.47 & 7.33 & 7.80 & 17.25 & 8.65 & 5.32 & 5.53 & 7.91 & 10.58 & 3.29 & 5.52 & 13.15 & 7.09 \\
\hline & PCV (\%) & 18.89 & 20.69 & 25.14 & 22.77 & 16.81 & 17.91 & 24.45 & 19.84 & 14.16 & 15.13 & 19.52 & 18.27 & 16.28 & 15.66 & 28.54 & 18.32 \\
\hline & Mean & 256 & 241 & 219 & 218 & 243 & 227 & 208 & 243 & 223 & 209 & 186 & 180 & 237 & 223 & 180 & 189 \\
\hline & Min. & 188 & 174 & 146 & 151 & 184 & 168 & 136 & 184 & 169 & 156 & 132 & 120 & 170 & 158 & 123 & 123 \\
\hline & Max. & 355 & 342 & 319 & 319 & 342 & 326 & 303 & 342 & 262 & 249 & 262 & 225 & 310 & 295 & 303 & 261 \\
\hline & CD at 0.05 & 73 & 81 & 72 & 81 & 74 & 74 & 73 & 74 & 59 & 59 & 67 & 54 & 76 & 66 & 92 & 64 \\
\hline & CV \% & 14 & 17 & 16 & 18 & 15 & 16 & 17 & 18 & 13 & 14 & 18 & 15 & 16 & 15 & 25 & 17 \\
\hline
\end{tabular}


Table.2 Genetic parameters of $50 \mathrm{~F}_{3}$ progenies selected from $\mathrm{F}_{2}$ population Cross- II (BG102/Naveen) based on different selection indices and grown under different crop ecology

\begin{tabular}{|c|c|c|c|c|c|c|c|c|c|c|c|c|c|c|c|c|c|}
\hline \multirow[t]{3}{*}{ Characters } & \multirow{3}{*}{$\begin{array}{c}\text { Genetic } \\
\text { parameters }\end{array}$} & \multicolumn{4}{|c|}{$\mathrm{I}^{\text {st }}$ method of selection (HY) } & \multicolumn{4}{|c|}{$2^{\text {nd }}$ method of selection (LY) } & \multicolumn{4}{|c|}{$3^{\text {rd }}$ method of selection (MTL) } & \multicolumn{4}{|c|}{$4^{\text {th }}$ method of selection (RPS) } \\
\hline & & \multicolumn{2}{|c|}{ I $^{\text {st }}$ Date } & \multicolumn{2}{|c|}{$2^{\text {nd }}$ Date } & \multicolumn{2}{|c|}{$\mathbf{I}^{\text {st }}$ Date } & \multicolumn{2}{|c|}{$2^{\text {nd }}$ Date } & \multicolumn{2}{|c|}{ I $^{\text {st }}$ Date } & \multicolumn{2}{|c|}{$2^{\text {nd }}$ Date } & \multicolumn{2}{|c|}{ I $^{\text {st }}$ Date } & \multicolumn{2}{|c|}{$2^{\text {nd }}$ Date } \\
\hline & & DS & $\mathbf{T P}$ & DS & TP & DS & TP & DS & TP & DS & TP & DS & TP & DS & TP & DS & TP \\
\hline \multirow[t]{9}{*}{ DFF } & $h^{2}(\%)$ & 68 & 64 & 24.3 & 77 & 61.05 & 65.91 & 50.17 & 43.31 & 3.06 & 45.02 & 9.85 & 95.72 & 53.34 & 65.94 & 32.59 & 55.04 \\
\hline & GA $(\%)$ & 4.8 & 4.3 & 2.06 & 4.4 & 4.28 & 4.17 & 3.69 & 2.65 & 0.11 & 1.42 & 0.45 & 26.92 & 2.67 & 3.18 & 1.94 & 2.75 \\
\hline & $\operatorname{GCV}(\%)$ & 2.9 & 2.6 & 1.94 & 2.5 & 2.68 & 2.41 & 2.34 & 1.99 & 0.4 & 1.13 & 0.83 & 14.33 & 2.24 & 2.09 & 1.97 & 1.88 \\
\hline & PCV (\%) & 3.6 & 3.2 & 3.93 & 2.9 & 3.43 & 2.96 & 3.3 & 3.03 & 2.3 & 1.69 & 2.66 & 14.65 & 3.07 & 2.58 & 3.45 & 2.53 \\
\hline & Mean & 75 & 85 & 79 & 90 & 75 & 86 & 79 & 90 & 73 & 76 & 83 & 88 & 73 & 77 & 83 & 88 \\
\hline & Min. & 70 & 80 & 74 & 85 & 72 & 82 & 76 & 87 & 71 & 73 & 82 & 86 & 69 & 72 & 80 & 84 \\
\hline & Max. & 82 & 93 & 86 & 97 & 81 & 91 & 85 & 96 & 76 & 80 & 87 & 91 & 79 & 83 & 90 & 94 \\
\hline & CD at 0.05 & 3 & 3 & 5 & 2 & 3 & 3 & 4 & 4 & 3 & 2 & 4 & 5 & 3 & 3 & 4 & 3 \\
\hline & $\mathrm{CV} \%$ & 2 & 2 & 3 & 1 & 2 & 2 & 2 & 2 & 2 & 1 & 3 & 3 & 2 & 2 & 3 & 2 \\
\hline \multirow[t]{9}{*}{ PH (cm) } & $h^{2}(\%)$ & 17 & 16 & 14.9 & 10 & 15.95 & 12.03 & 11.02 & 17.08 & 10.75 & 23.14 & 13.55 & 60.23 & 6.53 & 5.71 & 14.2 & 12.15 \\
\hline & GA (\%) & 2.2 & 2.1 & 2 & 1.1 & 2.21 & 1.5 & 1.46 & 1.67 & 1.35 & 2.88 & 2.18 & 20.53 & 0.75 & 0.63 & 1.59 & 1.12 \\
\hline & GCV $(\%)$ & 2.6 & 2.6 & 2.51 & 1.6 & 2.69 & 2.09 & 2.13 & 1.96 & 1.99 & 2.9 & 2.87 & 13.1 & 1.42 & 1.27 & 2.04 & 1.55 \\
\hline & PCV (\%) & 6.2 & 6.6 & 6.51 & 4.9 & 6.75 & 6.03 & 6.44 & 4.75 & 6.09 & 6.03 & 7.79 & 16.88 & 5.57 & 5.34 & 5.43 & 4.46 \\
\hline & Mean & 117 & 117 & 116 & 117 & 115 & 114 & 116 & 117 & 104 & 93 & 92 & 92 & 115 & 115 & 114 & 114 \\
\hline & Min. & 103 & 108 & 107 & 104 & 97 & 102 & 100 & 104 & 98 & 80 & 80 & 85 & 99 & 102 & 105 & 104 \\
\hline & Max. & 130 & 136 & 135 & 129 & 128 & 132 & 131 & 129 & 121 & 105 & 103 & 100 & 125 & 130 & 131 & 126 \\
\hline & CD at 0.05 & 13 & 15 & 15 & 11 & 15 & 14 & 15 & 10 & 13 & 10 & 14 & 19 & 13 & 12 & 12 & 10 \\
\hline & CV \% & 6 & 6 & 6 & 5 & 6 & 6 & 6 & 4 & 6 & 5 & 7 & 11 & 5 & 5 & 5 & 4 \\
\hline \multirow[t]{9}{*}{ PPP } & $h^{2}(\%)$ & 13 & 12 & 20.3 & 24 & 10.29 & 56.15 & 19.21 & 36.13 & 11.93 & 28.85 & 11.32 & 39.68 & 14.29 & 29.6 & 18.95 & 27.98 \\
\hline & GA $(\%)$ & 4.3 & 3.3 & 6.47 & 8.6 & 3.52 & 15.17 & 7.11 & 11.96 & 5.48 & 10.65 & 5.2 & 11.45 & 3.94 & 7.59 & 6.26 & 7.61 \\
\hline & $\operatorname{GCV}(\%)$ & 5.7 & 4.4 & 8.65 & 8.4 & 5.18 & 10.13 & 7.64 & 9.89 & 7.13 & 8.74 & 4.77 & 16.16 & 5.2 & 6.32 & 6.98 & 6.58 \\
\hline & PCV (\%) & 16 & 13 & 19.2 & 17 & 16.14 & 13.52 & 17.44 & 16.46 & 20.65 & 16.28 & 14.19 & 25.66 & 13.77 & 11.62 & 16.03 & 12.45 \\
\hline & Mean & 9 & 10 & 8 & 8 & 8 & 8 & 8 & 1 & 9 & 7 & 9 & 8 & 8 & 9 & 9 & 9 \\
\hline & Min. & 8 & 8 & 6 & 6 & 7 & 6 & 6 & 6 & 6 & 7 & 12 & 6 & 6 & 7 & 5 & 7 \\
\hline & Max. & 12 & 13 & 12 & 11 & 11 & 11 & 11 & 11 & 12 & 13 & 21 & 12 & 9 & 10 & 11 & 11 \\
\hline & CD at 0.05 & 3 & 2 & 3 & 2 & 3 & 1 & 3 & 2 & 3 & 4 & 3 & 3 & 2 & 2 & 2 & 2 \\
\hline & CV \% & 15 & 12 & 17 & 15 & 15 & 9 & 16 & 13 & 19 & 13 & 14 & 20 & 13 & 14 & 10 & 11 \\
\hline \multirow[t]{2}{*}{ GPP } & $h^{2}(\%)$ & 52 & 40 & 39.7 & 36 & 18.22 & 57.45 & 20.14 & 19.28 & 26.57 & 25.39 & 19.47 & 45.58 & 47.41 & 40.39 & 56.89 & 46.98 \\
\hline & GA (\%) & 19 & 15 & 17.2 & 12 & 6.53 & 19.78 & 7.63 & 7.03 & 8.19 & 7.94 & 6.3 & 20.13 & 19.6 & 15.18 & 23.26 & 16.32 \\
\hline
\end{tabular}




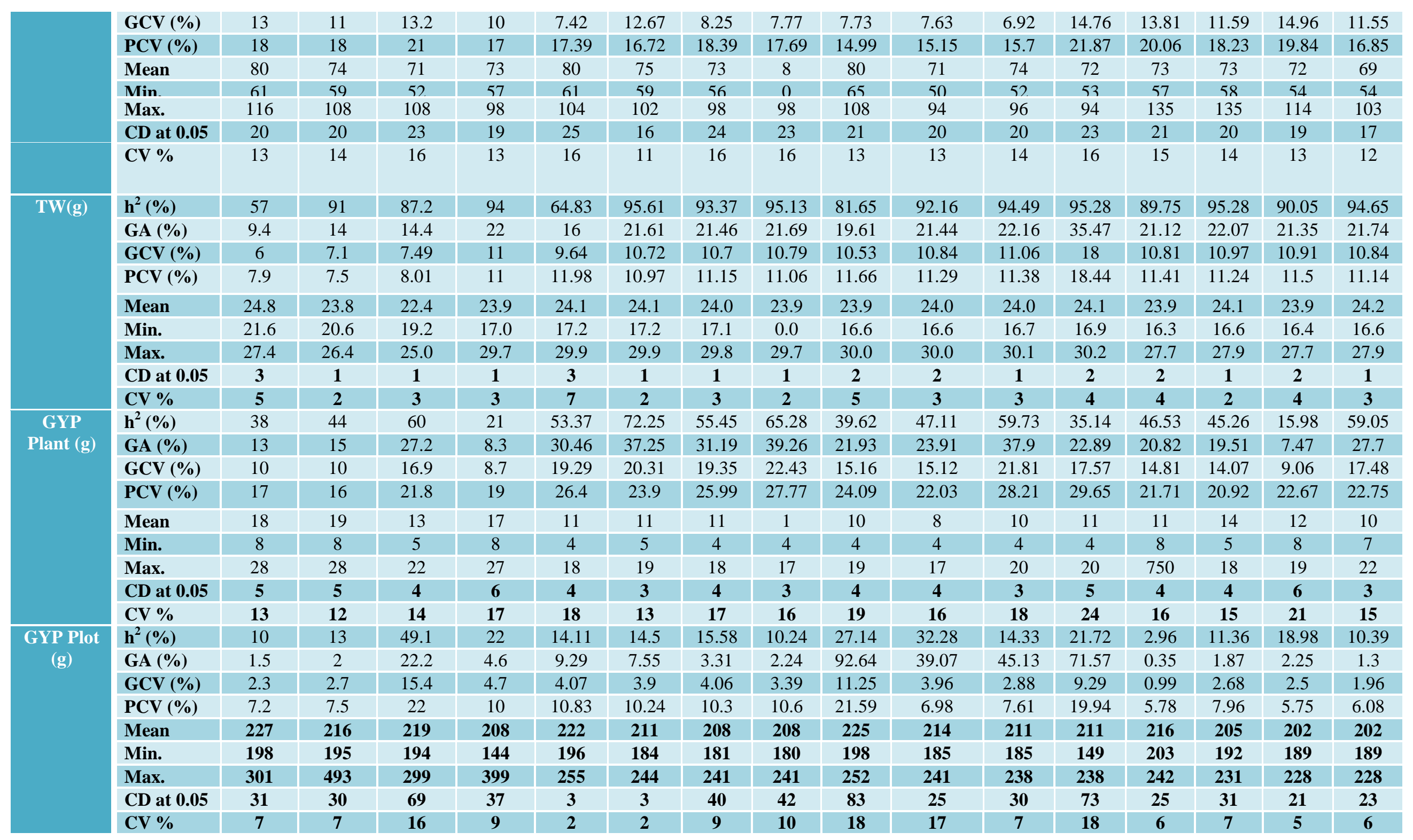


Table.3 Genetic parameters of $50 \mathrm{~F}_{3}$ progenies selected from $\mathrm{F}_{2}$ population Cross- III (BG102/ Reshmi $\left(\mathrm{F}_{1}\right)$ Commercial hybrid based from different selection indices and grown under different crop ecology

\begin{tabular}{|c|c|c|c|c|c|c|c|c|c|c|c|c|c|c|c|c|c|}
\hline \multirow[t]{3}{*}{ Characters } & \multirow{3}{*}{$\begin{array}{c}\text { Genetic } \\
\text { parameters }\end{array}$} & \multicolumn{4}{|c|}{$\mathrm{I}^{\mathrm{st}}$ method of selection (HY) } & \multicolumn{4}{|c|}{$2^{\text {nd }}$ method of selection (LY) } & \multicolumn{4}{|c|}{$3^{\text {rd }}$ method of selection (MTL) } & \multicolumn{4}{|c|}{$4^{\text {th }}$ method of selection (RPS) } \\
\hline & & \multicolumn{2}{|c|}{ I $^{\text {st }}$ Date } & \multicolumn{2}{|c|}{$2^{\text {nd }}$ Date } & \multicolumn{2}{|c|}{ I $^{\text {st }}$ Date } & \multicolumn{2}{|c|}{$2^{\text {nd }}$ Date } & \multicolumn{2}{|c|}{ I $^{\text {st }}$ Date } & \multicolumn{2}{|c|}{$2^{\text {nd }}$ Date } & \multicolumn{2}{|c|}{ I $^{\text {st }}$ Date } & \multicolumn{2}{|c|}{$2^{\text {nd }}$ Date } \\
\hline & & DS & $\mathbf{T P}$ & DS & $\mathbf{T P}$ & DS & $\mathbf{T P}$ & DS & $\mathbf{T P}$ & DS & $\mathbf{T P}$ & DS & $\mathbf{T P}$ & DS & $\mathbf{T P}$ & DS & $\mathbf{T P}$ \\
\hline \multirow[t]{9}{*}{ DFF } & $\mathbf{h}^{2}(\%)$ & 87.00 & 69.17 & 69.17 & 44.48 & 69.63 & 69.63 & 71.68 & 69.63 & 99.82 & 99.87 & 99.76 & 99.92 & 99.62 & 99.80 & 99.43 & 99.84 \\
\hline & GA (\%) & 11.53 & 8.56 & 9.01 & 4.08 & 8.25 & 7.31 & 7.89 & 6.84 & 84.32 & 84.14 & 84.15 & 84.07 & 86.12 & 85.88 & 85.82 & 85.69 \\
\hline & $\operatorname{GCV}(\%)$ & 5.99 & 4.99 & 5.25 & 3.06 & 4.80 & 4.25 & 4.52 & 3.97 & 48.77 & 48.65 & 48.69 & 48.60 & 48.74 & 48.64 & 48.65 & 48.59 \\
\hline & $\operatorname{PCV}(\%)$ & 6.41 & 6.01 & 6.32 & 4.59 & 5.75 & 5.10 & 5.34 & 4.76 & 48.81 & 48.68 & 48.74 & 48.62 & 48.84 & 48.69 & 48.78 & 48.63 \\
\hline & Mean & 81 & 91 & 87 & 97 & 77 & 87 & 83 & 93 & 81 & 91 & 86 & 97 & 79 & 89 & 84 & 95 \\
\hline & Min. & 75 & 83 & 79 & 89 & 72 & 82 & 78 & 88 & 72 & 82 & 77 & 88 & 72 & 82 & 77 & 88 \\
\hline & Max. & 90 & 100 & 95 & 106 & 87 & 97 & 93 & 103 & 89 & 99 & 95 & 105 & 89 & 99 & 95 & 105 \\
\hline & CD at 0.05 & 4 & 6 & 6 & 6 & 5 & 5 & 5 & 5 & 3 & 3 & 3 & 2 & 4 & 3 & 5 & 3 \\
\hline & $\mathrm{CV} \%$ & 2 & 3 & 4 & 3 & 3 & 3 & 3 & 3 & 2 & 2 & 2 & 1 & 3 & 2 & 4 & 2 \\
\hline \multirow[t]{9}{*}{ PH (cm) } & $\mathbf{h}^{2}(\%)$ & 82.50 & 36.72 & 5.08 & 15.57 & 55.20 & 25.87 & 43.13 & 28.08 & 97.06 & 94.82 & 95.39 & 95.73 & 91.77 & 94.87 & 95.65 & 95.14 \\
\hline & GA (\%) & 18.16 & 7.19 & 1.23 & 2.80 & 15.45 & 5.91 & 13.08 & 6.64 & 85.96 & 82.82 & 85.22 & 83.61 & 77.95 & 79.44 & 74.72 & 79.69 \\
\hline & $\operatorname{GCV}(\%)$ & 12.51 & 5.75 & 2.65 & 3.44 & 10.09 & 5.64 & 9.66 & 6.07 & 50.41 & 49.14 & 50.42 & 49.37 & 49.93 & 49.15 & 50.46 & 49.30 \\
\hline & PCV (\%) & 13.77 & 9.50 & 11.77 & 8.73 & 13.58 & 11.09 & 14.71 & 11.47 & 51.17 & 50.47 & 51.62 & 50.47 & 52.12 & 50.46 & 51.59 & 50.54 \\
\hline & Mean & 80 & 90 & 82 & 117 & 81 & 92 & 81 & 89 & 83 & 91 & 83 & 88 & 83 & 94 & 84 & 92 \\
\hline & Min. & 51 & 72 & 67 & 104 & 54 & 68 & 54 & 65 & 59 & 69 & 60 & 66 & 55 & 81 & 81 & 78 \\
\hline & Max. & 95 & 103 & 97 & 138 & 103 & 109 & 103 & 106 & 109 & 110 & 110 & 107 & 110 & 110 & 110 & 107 \\
\hline & CD at 0.05 & 15 & 14 & 19 & 20 & 15 & 18 & 18 & 17 & 12 & 18 & 16 & 16 & 21 & 18 & 15 & 17 \\
\hline & CV \% & 9 & 8 & 11 & 8 & 9 & 10 & 11 & 10 & 9 & 11 & 11 & 10 & 15 & 11 & 11 & 11 \\
\hline \multirow[t]{9}{*}{ PPP } & $h^{2}(\%)$ & 87.48 & 38.56 & 22.62 & 40.79 & 39.55 & 36.17 & 37.64 & 28.92 & 68.04 & 17.49 & 64.66 & 78.08 & 73.05 & 89.54 & 71.95 & 79.57 \\
\hline & GA $(\%)$ & 12.04 & 14.26 & 8.38 & 20.74 & 9.65 & 12.08 & 15.78 & 12.40 & 70.67 & 19.70 & 60.39 & 73.94 & 71.63 & 81.14 & 67.60 & 77.24 \\
\hline & GCV $(\%)$ & 13.75 & 11.35 & 8.90 & 9.99 & 10.02 & 12.76 & 12.35 & 11.55 & 45.43 & 27.83 & 44.93 & 47.58 & 46.39 & 49.58 & 46.38 & 47.84 \\
\hline & PCV (\%) & 14.70 & 18.28 & 18.71 & 15.64 & 15.94 & 21.22 & 20.13 & 21.47 & 55.08 & 66.55 & 55.87 & 53.85 & 54.28 & 52.39 & 54.68 & 53.62 \\
\hline & Mean & 8 & 8 & 8 & 8 & 8 & 7 & 7 & 7 & 7 & 7 & 7 & 7 & 7 & 7 & 7 & 7 \\
\hline & Min. & 1 & 5 & 3 & 6 & 3 & 6 & 4 & 4 & 5 & 5 & 4 & 5 & 5 & 5 & 5 & 5 \\
\hline & Max. & 11 & 11 & 10 & 16 & 14 & 13 & 10 & 10 & 9 & 9 & 9 & 9 & 12 & 11 & 11 & 11 \\
\hline & CD at 0.05 & 2 & 2 & 3 & 3 & 2 & 2 & 2 & 2 & 4 & 7 & 4 & 3 & 4 & 2 & 3 & 3 \\
\hline & CV \% & 14 & 14 & 16 & 12 & 12 & 17 & 16 & 18 & 31 & 60 & 33 & 25 & 28 & 17 & 29 & 24 \\
\hline \multirow{2}{*}{ GPP } & $\mathbf{h}^{2}(\%)$ & 87.02 & 23.52 & 46.49 & 49.25 & 36.35 & 20.55 & 39.10 & 26.00 & 88.40 & 87.76 & 81.34 & 84.15 & 86.38 & 84.86 & 88.90 & 86.43 \\
\hline & GA (\%) & 19.67 & 7.37 & 18.65 & 20.49 & 12.57 & 6.53 & 13.25 & 8.08 & 83.88 & 78.47 & 77.12 & 76.02 & 82.92 & 75.73 & 81.75 & 75.68 \\
\hline
\end{tabular}




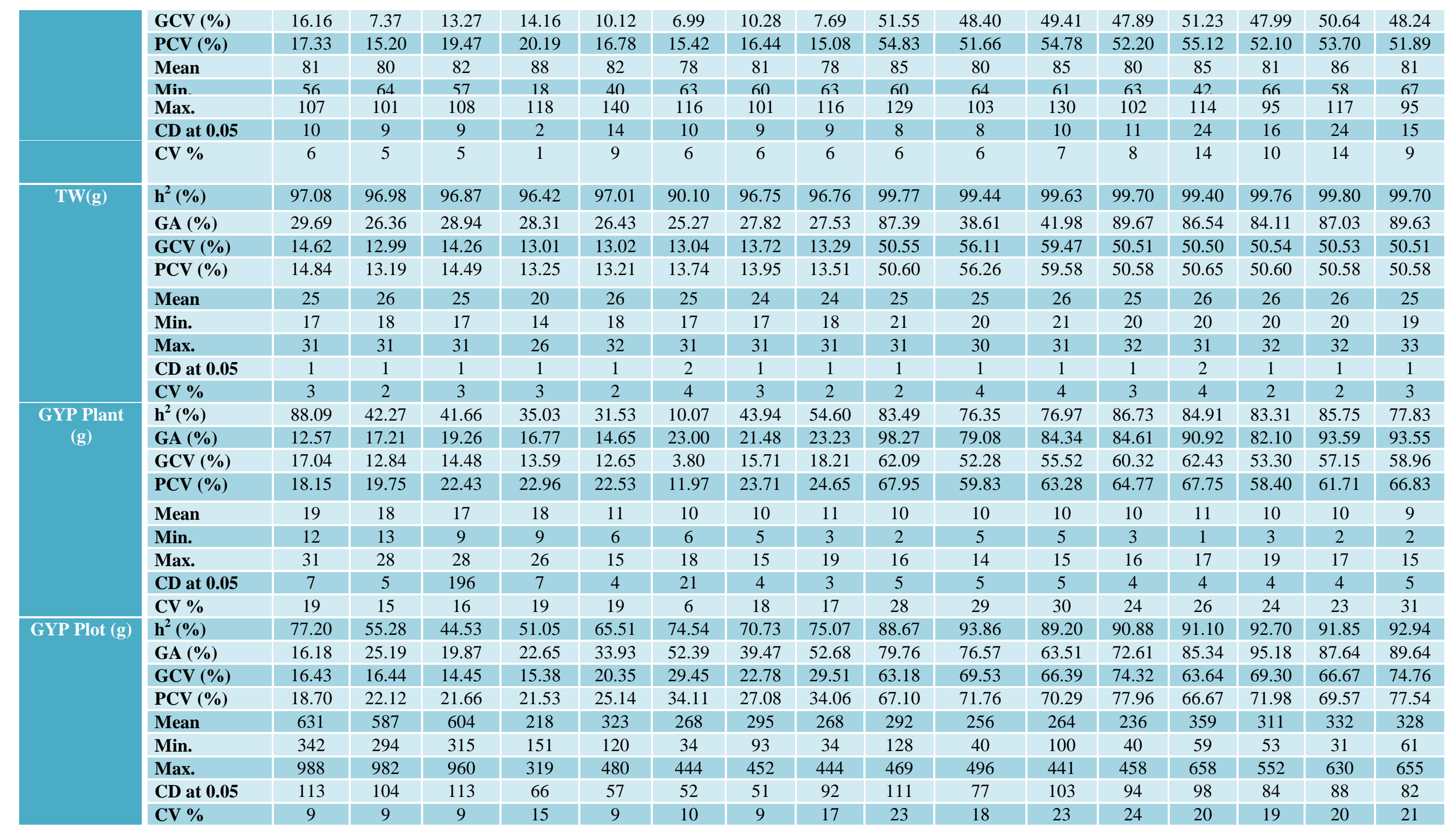


Table.4 Estimates of standardized selection differential, standardized selection response and realized heritability for different traits from two methods as well as two date of sowing based on different methods of selection of Cross- I (BG102/BPT5204)

\begin{tabular}{|c|c|c|c|c|c|c|c|c|c|c|c|c|c|c|c|c|c|c|}
\hline \multirow{3}{*}{$\begin{array}{l}\text { Methods of } \\
\text { selection and } \\
\text { selection } \\
\text { intensity } \\
(5 \%)\end{array}$} & \multirow{3}{*}{ 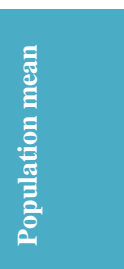 } & \multirow{3}{*}{ 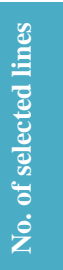 } & \multirow{3}{*}{ 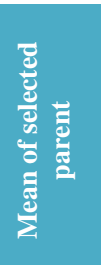 } & \multirow{3}{*}{ 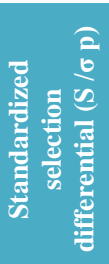 } & \multicolumn{4}{|c|}{ Progeny mean } & \multicolumn{4}{|c|}{$\begin{array}{l}\text { Standardized selection } \\
\text { response }(\mathrm{R} / \sigma \mathrm{p})\end{array}$} & \multirow{3}{*}{ 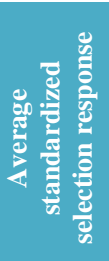 } & \multicolumn{4}{|c|}{ Realized heritability (R/S) } & \multirow{3}{*}{ 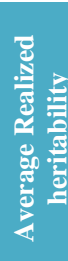 } \\
\hline & & & & & \multicolumn{2}{|c|}{$I^{\text {st }}$ Date } & \multicolumn{2}{|c|}{$2^{\text {nd }}$ Date } & \multicolumn{2}{|c|}{$I^{\text {st }}$ Date } & \multicolumn{2}{|c|}{$2^{\text {nd }}$ Date } & & \multicolumn{2}{|c|}{$I^{\text {st }}$ Date } & \multicolumn{2}{|c|}{$2^{\text {nd }}$ Date } & \\
\hline & & & & & DS & TP & DS & TP & DS & TP & DS & TP & & DS & $\mathbf{T P}$ & DS & TP & \\
\hline Based on HY & & 50 & & & & & & & & & & & & & & & & \\
\hline PH (cm) & 116.15 & & 122.12 & 0.46 & 122.01 & 121.06 & 121.79 & 121.70 & 0.45 & 0.38 & 0.43 & 0.43 & 0.42 & 0.98 & 0.82 & 0.94 & 0.93 & 0.92 \\
\hline $\begin{array}{l}\text { PPP } \\
\end{array}$ & 8.08 & & 12.32 & 1.41 & 12.07 & 10.63 & 11.00 & 12.29 & 1.33 & 0.85 & 0.97 & 1.40 & 1.14 & 0.94 & 0.60 & 0.69 & 0.99 & 0.81 \\
\hline GPP & 78.48 & & 109.48 & 1.14 & 80.95 & 103.34 & 99.22 & 88.37 & 0.09 & 0.92 & 0.76 & 0.36 & 0.53 & 0.08 & 0.80 & 0.67 & 0.32 & 0.47 \\
\hline $\begin{array}{l}\text { GYP Plant } \\
(\mathrm{g})\end{array}$ & 10.12 & & 25.38 & 2.68 & 24.90 & 24.52 & 20.25 & 17.49 & 2.60 & 2.53 & 1.78 & 1.30 & 2.05 & 0.97 & 0.94 & 0.66 & 0.48 & 0.76 \\
\hline Based on LY & & 50 & & & & & & & & & & & & & & & & \\
\hline PH (cm) & 116.15 & & 122.34 & 0.48 & 122.30 & 121.43 & 122.09 & 122.20 & 0.47 & 0.41 & 0.46 & 0.46 & 0.45 & 0.99 & 0.85 & 0.96 & 0.98 & 0.95 \\
\hline PPP & 8.07 & & 10.06 & 0.66 & 9.74 & 9.94 & 10.00 & 9.74 & 0.55 & 0.62 & 0.64 & 0.55 & 0.59 & 0.84 & 0.94 & 0.97 & 0.84 & 0.90 \\
\hline GPP & 78.48 & & 89.76 & 0.42 & 88.06 & 89.30 & 89.59 & 89.00 & 0.35 & 0.40 & 0.41 & 0.39 & 0.39 & 0.85 & 0.96 & 0.98 & 0.93 & 0.93 \\
\hline $\begin{array}{l}\text { GYP Plant } \\
\text { (g) }\end{array}$ & 10.12 & & 16.43 & 1.11 & 15.90 & 15.50 & 15.72 & 15.90 & 1.02 & 0.95 & 0.98 & 1.02 & 0.99 & 0.92 & 0.85 & 0.89 & 0.92 & 0.90 \\
\hline $\begin{array}{l}\text { Based on } \\
\text { MTL }\end{array}$ & & 48 & & & & & & & & & & & & & & & & \\
\hline PH (cm) & 116.15 & & 101.92 & -1.09 & 114.72 & 115.37 & 115.62 & 115.33 & $\overline{-}$ & $\overline{-}$ & $\overline{-}$ & $\overline{-}$ & -0.07 & 0.10 & 0.05 & 0.04 & 0.06 & 0.06 \\
\hline PPP & 8.07 & & 9.08 & 0.34 & 9.00 & 8.63 & 8.78 & 8.45 & 0.31 & 0.19 & 0.24 & 0.13 & 0.22 & 0.92 & 0.55 & 0.70 & 0.38 & 0.64 \\
\hline GPP & 78.48 & & 87.85 & 0.35 & 85.19 & 85.96 & 87.80 & 84.48 & 0.25 & 0.28 & 0.34 & 0.22 & 0.27 & 0.72 & 0.80 & 0.99 & 0.64 & 0.79 \\
\hline $\begin{array}{l}\text { GYP Plant } \\
\text { (g) }\end{array}$ & 10.12 & & 10.76 & 0.11 & 10.72 & 10.43 & 10.69 & 10.63 & 0.11 & 0.05 & 0.10 & 0.09 & 0.09 & 0.94 & 0.48 & 0.89 & 0.79 & 0.78 \\
\hline $\begin{array}{l}\text { Based on } \\
\text { RPS }\end{array}$ & & 50 & & & & & & & & & & & & & & & & \\
\hline PH (cm) & 116.15 & & 124.72 & 0.66 & 120.66 & 122.90 & 123.96 & 123.88 & 0.35 & 0.52 & 0.60 & 0.59 & 0.52 & 0.53 & 0.79 & 0.91 & 0.90 & 0.78 \\
\hline$\overline{\text { PPP }}$ & 8.07 & & 9.72 & 0.55 & 9.65 & 8.83 & 9.28 & 9.36 & 0.52 & 0.25 & 0.40 & 0.43 & 0.40 & 0.96 & 0.46 & 0.74 & 0.78 & 0.74 \\
\hline GPP & 78.48 & & 90.28 & 0.43 & 88.74 & 82.61 & 88.56 & 89.03 & 0.38 & 0.15 & 0.37 & 0.39 & 0.32 & 0.87 & 0.35 & 0.85 & 0.89 & 0.74 \\
\hline $\begin{array}{l}\text { GYP Plant } \\
\text { (g) }\end{array}$ & 10.12 & & 16.08 & 1.05 & 15.88 & 15.58 & 14.82 & 14.82 & 1.01 & 0.96 & 0.83 & 0.83 & 0.91 & 0.97 & 0.92 & 0.79 & 0.79 & 0.87 \\
\hline
\end{tabular}


Table.5 Estimates of Standardized selection differential, standardized selection response and realized heritability for different traits from two methods as well as two date of sowing based on different methods of selection of Cross- II (BG102/Naveen)

\begin{tabular}{|c|c|c|c|c|c|c|c|c|c|c|c|c|c|c|c|c|c|c|}
\hline \multirow{3}{*}{$\begin{array}{c}\text { Methods of } \\
\text { selection and } \\
\text { selection } \\
\text { intensity } \\
(5 \%)\end{array}$} & \multirow{3}{*}{ 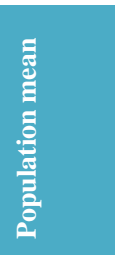 } & \multirow{3}{*}{ 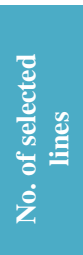 } & \multirow{3}{*}{ 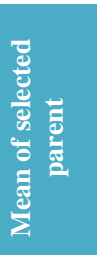 } & \multirow{3}{*}{ 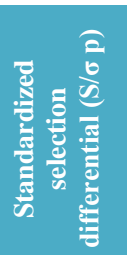 } & \multicolumn{4}{|c|}{ Progeny mean } & \multicolumn{4}{|c|}{$\begin{array}{l}\text { Standardized selection response } \\
(\mathbf{R} / \sigma p)\end{array}$} & \multirow{3}{*}{ 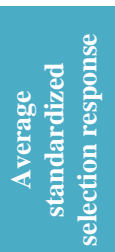 } & \multicolumn{4}{|c|}{ Realized heritability (R/S) } & \multirow{3}{*}{ 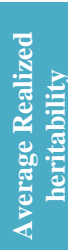 } \\
\hline & & & & & \multicolumn{2}{|c|}{ I $^{\text {st }}$ Date } & \multicolumn{2}{|c|}{$2^{\text {nd }}$ Date } & \multicolumn{2}{|c|}{$I^{\text {st }}$ Date } & \multicolumn{2}{|c|}{$2^{\text {nd }}$ Date } & & \multicolumn{2}{|c|}{$I^{\text {st }}$ Date } & \multicolumn{2}{|c|}{$2^{\text {nd }}$ Date } & \\
\hline & & & & & DS & TP & DS & $\mathbf{T P}$ & DS & $\mathbf{T P}$ & DS & TP & & DS & TP & DS & TP & \\
\hline Based on HY & & 50 & & & & & & & & & & & & & & & & \\
\hline PH (cm) & 115.36 & & 117.52 & 77.88 & 117.10 & 116.70 & 115.90 & 117.00 & 77.45 & 77.06 & 76.26 & 77.36 & 77.03 & 0.99 & 0.99 & 0.98 & 0.99 & 0.99 \\
\hline PPP & 7.57 & & 9.98 & 7.14 & 9.29 & 9.74 & 7.86 & 7.96 & 6.46 & 6.91 & 5.02 & 5.12 & 5.88 & 0.90 & 0.97 & 0.70 & 0.72 & 0.82 \\
\hline GPP & 69.6 & & 80.58 & 67.47 & 80.23 & 74.27 & 71.01 & 73.38 & 67.12 & 61.16 & 57.90 & 60.28 & 61.62 & 0.99 & 0.91 & 0.86 & 0.89 & 0.91 \\
\hline $\begin{array}{l}\text { GYP Plant } \\
\text { (g) }\end{array}$ & 10.01 & & 23.06 & 21.17 & 18.31 & 18.62 & 12.97 & 17.38 & 16.42 & 16.74 & 11.08 & 15.50 & 14.94 & 0.78 & 0.79 & 0.52 & 0.73 & 0.71 \\
\hline Based on LY & & 50 & & & & & & & & & & & & & & & & \\
\hline PH $(\mathrm{cm})$ & 115.36 & & 118.20 & 78.56 & 115.00 & 113.66 & 115.60 & 117.00 & 75.36 & 74.02 & 75.96 & 77.36 & 75.68 & 0.96 & 0.94 & 0.97 & 0.98 & 0.96 \\
\hline PPP & 7.57 & & 8.32 & 5.48 & 8.18 & 7.92 & 8.16 & 7.96 & 5.34 & 5.09 & 5.32 & 5.12 & 5.22 & 0.97 & 0.93 & 0.97 & 0.93 & 0.95 \\
\hline GPP & 69.6 & & 81.82 & 68.71 & 80.00 & 74.97 & 72.59 & 73.30 & 66.90 & 61.87 & 59.48 & 60.19 & 62.11 & 0.97 & 0.90 & 0.87 & 0.88 & 0.91 \\
\hline $\begin{array}{l}\text { GYP Plant } \\
\text { (g) }\end{array}$ & 10.01 & & 14.17 & 12.28 & 10.82 & 11.30 & 10.89 & 9.89 & 8.94 & 9.42 & 9.00 & 8.01 & 8.84 & 0.73 & 0.77 & 0.73 & 0.65 & 0.72 \\
\hline $\begin{array}{l}\text { Based on } \\
\text { MTL }\end{array}$ & & 49 & & & & & & & & & & & & & & & & \\
\hline PH (cm) & 115.36 & & 105.69 & 66.05 & 103.50 & 91.76 & 92.94 & 92.05 & 63.86 & 52.12 & 53.29 & 52.41 & 55.42 & 0.97 & 0.79 & 0.81 & 0.79 & 0.84 \\
\hline$\overline{\mathbf{P P P}}$ & 7.57 & & 9.14 & 6.31 & 8.58 & 7.44 & 8.82 & 8.30 & 5.75 & 4.60 & 5.98 & 5.46 & 5.45 & 0.91 & 0.73 & 0.95 & 0.87 & 0.87 \\
\hline GPP & 69.6 & & 90.61 & 77.50 & 79.79 & 74.33 & 70.61 & 71.53 & 66.68 & 61.22 & 57.51 & 58.43 & 60.96 & 0.86 & 0.79 & 0.74 & 0.75 & 0.79 \\
\hline $\begin{array}{l}\text { GYP Plant } \\
\text { (g) }\end{array}$ & 10.01 & & 11.51 & 9.63 & 9.74 & 10.17 & 7.91 & 10.54 & 7.85 & 8.28 & 6.03 & 8.65 & 7.70 & 0.82 & 0.86 & 0.63 & 0.90 & 0.80 \\
\hline $\begin{array}{l}\text { Based on } \\
\text { RPS }\end{array}$ & & 50 & & & & & & & & & & & & & & & & \\
\hline PH (cm) & 115.36 & & 115.76 & 76.12 & 115.37 & 113.58 & 115.06 & 114.48 & 75.72 & 73.94 & 75.42 & 74.84 & 74.98 & 0.99 & 0.97 & 0.99 & 0.98 & 0.98 \\
\hline PPP & 7.57 & & 8.76 & 5.92 & 8.05 & 8.62 & 8.60 & 9.11 & 5.22 & 5.78 & 5.77 & 6.28 & 5.76 & 0.88 & 0.98 & 0.97 & 1.06 & 0.97 \\
\hline GPP & 69.6 & & 73.42 & 60.31 & 72.90 & 72.26 & 72.61 & 69.32 & 59.79 & 59.15 & 59.50 & 56.21 & 58.66 & 0.99 & 0.98 & 0.99 & 0.93 & 0.97 \\
\hline $\begin{array}{l}\text { GYP Plant } \\
\text { (g) }\end{array}$ & 10.01 & & 14.14 & 12.25 & 11.35 & 11.83 & 14.03 & 10.40 & 9.47 & 9.95 & 12.15 & 8.51 & 10.02 & 0.77 & 0.81 & 0.99 & 0.69 & 0.82 \\
\hline
\end{tabular}


Table.6 Estimates of Standardized selection differential, standardized selection response and realized heritability for different traits from two methods as well as two Date of sowing based on different methods of selection of Cross- III(BG102/ Reshmi ( $\left.\left.F_{1}\right)\right)$

Commercial Pvt. hybrid

\begin{tabular}{|c|c|c|c|c|c|c|c|c|c|c|c|c|c|c|c|c|c|c|}
\hline \multirow{3}{*}{$\begin{array}{c}\text { Methods of } \\
\text { selection and } \\
\text { selection intensity } \\
(5 \%)\end{array}$} & \multirow{3}{*}{ 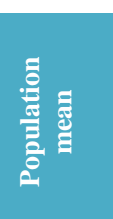 } & \multirow{3}{*}{ 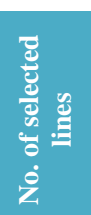 } & \multirow{3}{*}{ 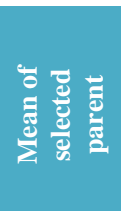 } & \multirow{3}{*}{ 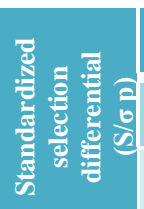 } & \multicolumn{4}{|c|}{ Progeny mean } & \multicolumn{4}{|c|}{$\begin{array}{l}\text { standardized selection response } \\
\text { (R/ } / \mathrm{p})\end{array}$} & \multirow{3}{*}{ 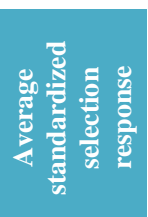 } & \multicolumn{4}{|c|}{ Realized heritability (R/S) } & \multirow{3}{*}{ 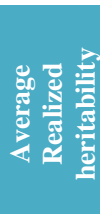 } \\
\hline & & & & & \multicolumn{2}{|c|}{$\mathrm{I}^{\mathrm{st}}$ Date of sowing } & \multicolumn{2}{|c|}{$\begin{array}{l}2^{\text {nd }} \text { Date of } \\
\text { sowing }\end{array}$} & \multicolumn{2}{|c|}{$\begin{array}{l}I^{\text {st }} \text { Date of } \\
\text { sowing }\end{array}$} & \multicolumn{2}{|c|}{$2^{\text {nd }}$ Date of sowing } & & \multicolumn{2}{|c|}{$\begin{array}{l}I^{\text {st }} \text { Date of } \\
\text { sowing }\end{array}$} & \multicolumn{2}{|c|}{$\begin{array}{l}2^{\text {nd }} \text { Date of } \\
\text { sowing }\end{array}$} & \\
\hline & & & & & DS & $\mathbf{T P}$ & DS & TP & DS & $\mathbf{T P}$ & DS & TP & & DS & TP & DS & TP & \\
\hline \multicolumn{19}{|l|}{ Based on HY } \\
\hline PH (cm) & 115 & 50 & 119.1 & 108.85 & 79.62 & 89.8 & 82.4 & 117.2 & 69.35 & 79.5 & 72.13 & 106.93 & 81.99 & 0.64 & 0.73 & 0.66 & 0.98 & 0.75 \\
\hline GPP & 80.2 & & 95.6 & 92.64 & 80.61 & 80.42 & 81.8 & 88.36 & 77.65 & 77.5 & 78.82 & 85.4 & 79.83 & 0.84 & 0.84 & 0.85 & 0.92 & 0.86 \\
\hline GYP Plant (g) & 25.3 & & 19.8 & 17.46 & 19.17 & 17.56 & 16.8 & 17.61 & 16.83 & 15.2 & 14.45 & 15.27 & 15.44 & 0.96 & 0.87 & 0.83 & 0.87 & 0.88 \\
\hline Based on LY & & 50 & & & & & & & & & & & & & & & & \\
\hline$\overline{\mathrm{PH}}(\mathrm{cm})$ & 115 & & 115.1 & 104.81 & 81.33 & 92.48 & 81.2 & 89.45 & 71.06 & 82.2 & 70.97 & 79.18 & 75.86 & 0.68 & 0.78 & 0.68 & 0.76 & 0.73 \\
\hline PPP & 6.82 & & 8.6 & 6.07 & 8.2 & 7 & 7.07 & 6.85 & 5.67 & 4.47 & 4.54 & 4.32 & 4.75 & 0.93 & 0.74 & 0.75 & 0.71 & 0.78 \\
\hline GPP & 80.2 & & 85.82 & 82.86 & 82.04 & 78.47 & 80.7 & 78.47 & 79.08 & 75.5 & 77.77 & 75.51 & 76.97 & 0.95 & 0.91 & 0.94 & 0.91 & 0.93 \\
\hline Based on MTL & & 42 & & & & & & & & & & & & & & & & \\
\hline$\overline{\mathrm{PH}}(\mathbf{c m})$ & 115 & & 101.7 & 91.4 & 83.38 & 91.41 & 83.4 & 88.48 & 73.11 & 81.1 & 73.14 & 78.21 & 76.40 & 0.80 & 0.89 & 0.8 & 0.86 & 0.84 \\
\hline$\overline{\text { PPP }}$ & 6.82 & & 8 & 5.47 & 7.45 & 7.29 & 7.03 & 7.14 & 4.92 & 4.76 & 4.5 & 4.61 & 4.70 & 0.90 & 0.87 & 0.82 & 0.84 & 0.86 \\
\hline GPP & 80.2 & & 103.7 & 100.75 & 85.09 & 80.22 & 85.1 & 79.5 & 82.13 & 77.3 & 82.16 & 76.54 & 79.52 & 0.82 & 0.77 & 0.82 & 0.76 & 0.79 \\
\hline GYP Plant (g) & 25.3 & & 10.53 & 8.19 & 9.92 & 9.87 & 10.4 & 10.25 & 7.58 & 7.53 & 8.1 & 7.91 & 7.78 & 0.93 & 0.92 & 0.99 & 0.97 & 0.95 \\
\hline Based RPS & & 50 & & & & & & & & & & & & & & & & \\
\hline PH (cm) & 115 & & 119.7 & 109.45 & 83.38 & 91.41 & 83.4 & 88.48 & 73.11 & 81.1 & 73.14 & 78.21 & 76.40 & 0.67 & 0.74 & 0.67 & 0.71 & 0.7 \\
\hline$\overline{\mathbf{P P P}}$ & 6.82 & & 7.56 & 5.03 & 7.29 & 7.07 & 6.83 & 6.9 & 4.76 & 4.54 & 4.3 & 4.37 & 4.49 & 0.95 & 0.90 & 0.86 & 0.87 & 0.9 \\
\hline$\overline{\text { GPP }}$ & 80.2 & & 99.34 & 96.38 & 84.56 & 80.97 & 86.1 & 81.3 & 81.6 & 78 & 83.14 & 78.34 & 80.27 & 0.85 & 0.81 & 0.86 & 0.81 & 0.83 \\
\hline GYP Plant (g) & 25.3 & & 11.32 & 8.98 & 10.82 & 10.27 & 10.3 & 8.73 & 8.48 & 7.93 & 7.98 & 6.39 & 7.70 & 0.94 & 0.88 & 0.89 & 0.71 & 0.86 \\
\hline
\end{tabular}

$\mathrm{DFF}=$ Days to $50 \%$ Flowering, $\mathrm{PH}=$ Plant Height, $\mathrm{PPP}=$ Panicle per Plant, $\mathrm{PL}=$ Panicle Length, GPP $=$ Grains per Panicle, GYP plant $=$ Grain yield per plant, GYP plot $=$ Grain yield per plot, HY $=$ High Yielder, LY $=$ Low yielder, MTL $=$ Multi trait limit, RPS $=$ Random plant selection, DS $=$ Direct seeding, TP $=$ Transplanting, GCV = Genotypic coefficient of variation, PCV = Phenotypic coefficient of variation and GA = Genetic Advance. 
While, Twin all methods and both date of planting indicating the preponderance of additive gene action was exhibited high $\mathrm{h}^{2}$ coupled with high GA as per cent of mean earlier finding reported by Lokprakash et al., (1992) and Lingaiah (2015), Singh et al., (2013) and Tuhina et al., (2015). The high heritable characters should be fairly easy and could be used as a selection criterion for future hybridisation programme. This is because there would be close correspondence between the genotype and phenotype due to relatively smaller contribution of the environment to the phenotype. Heritability estimates along with genetic gain are normally more helpful in predicting the gain under selection rather than only heritability estimates (Johanson et al., 1955).

Cross-I had exhibited maximum average selection response for all the studied traits in LY followed by HY and RPS selection method. It might be probable that delaying selection reduces the frequency of high yielding genotypes resulting a greater frequency of low yielding genotypes. Cross II and Cross - III were obtained highest average selection response for all studied traits in HY followed by LY selection method are given in the (Table 4, 5 and 6). Although the results indicate that the improvement obtained by selecting in late generation is just as effective as in early generations, a strong argument for selecting for yield in early generations is to permit testing in many sites and years at an early stage. The results of selection for low yield supported by earlier findings Shebeshki L. H. 1967, Whan et al., (1982), Verma and Mani (2000), and Ahmad et al., (2017).

Cross -II exhibited highest standardized selection differential for all studied traits based on HY followed by LY selection method while in Cross - II as well as Cross III in HY followed by RPS methods. It might be due to the frequency of best genotypes rapidly decreases with advancing generation similar finding earlier reported by Shebeshki L. H. 1967, Verma and Mani (2000), and Ahmad et al., (2017).

In Cross -I; Selection based on HY, high realized $h^{2}$ was observed for all characters under different methods of planting in both dates of sowing except in $2^{\text {nd }}$ date of TP for traits GPP and GYP plant it might be due to environmental effects. High level of realized $\mathrm{h}^{2}$ was also recorded under RPS for all the traits in different methods of planting on different dates of sowing barring for the trait PH under DS condition while for PPP and GPP under TP condition in I $^{\text {st }}$ date of planting which were comprising low realized $h^{2}$ it might be due to the influence of environment, whereas under MTL for PH and PPP low $\mathrm{h}^{2}$ were recorded in different method of planting as well as on different date of sowing except in DS condition in $2^{\text {nd }}$ date of sowing for the traits PPP, while high realized $h^{2}$ was recorded for traits GPP and GYP plant in different method of planting on both dates of sowing except for TP on $\mathrm{I}^{\text {st }}$ of sowing of GPP whereas Cross -II and Cross -III were obtained high realised $h^{2}$ for all studied traits in different methods of planting on both dates of sowing suggesting that early generation of selection may be effective. This finding is corroborated with Verma and Mani (2000), Vir and Singh (2005) and Kumar et al., (2009) and Ahmad et al., (2017).

In the present investigation it can be concluded that among different methods of selection HY followed by LY selection method may be effective in early segregating generation for Cross - II as well as Cross - III whereas for Cross - I; LY followed by HY selection method may be more effective. It might be probable that delaying selection reduces the frequency of high yielding genotypes resulting in a greater frequency of 
low yielding genotypes. Although the results indicate that the improvement obtained by selecting in late generation is just as effective as in early generations, a strong argument for selecting for yield in early generations is to permit testing in many sites and years at an early stage. However, on the other hand judicious selection causes increase in mean of the population for certain traits. Since, breeder is concerned with the enhancing of means for the traits; he may get an improved population at the onset of homozygosity at $\mathrm{F}_{8}$ and onward stages.

\section{Acknowledgements}

Authors are highly thankful to BAU, Kanke, Ranch who has provided all facilities for conducting the experiment on Rice.

\section{References}

Ahmad, Mehboob, Iqbal, Mazhar, Ahmed, Khan, Bilal and Ullah, Khan, Zaheer (2017). Response to Selection and Decline in Variability, Heritabilty and Genetic Advance from $\mathrm{F}_{2}$ to $\mathrm{F}_{3}$ Generation of Tomato (Solanum lycopercicum). International Journal of Plant Research, 7(1): 1-4.

Allard, R.W. (1960).Principles of plant breeding. John Wiley and Sons. Inc., U.S.A. pp. 485.

Burton, G. W. (1952). Quantitative inheritance in pearl millet ( $P$. typhoides L.). Agron. J.50: 503.

Chakraborty, R. and Chakraborty, S. (2010). Genetic variability and correlation of some morphometric traits with grain yield in bold grained rice (Oryza sativa L.) gene pool of Barak valley. American Eurasian Journal of Sustainable Agriculture. 4(1): 26-29b.

Dutta, Pallabi, Dutta, Partha, Nath and Borua, P.K. (2013). Morphological Traits as Selection Indices in Rice: A Statistical
View. Universal Journal of Agricultural Research 1(3): 85-96.

Falconer D. S. (1989). Introduction to Quantitative Genetics.3rd edn. Longman, Burnt Mill.

Gala, Anis, Ayman, E.L. Sabagh, Abdelfatah, Ghareb and Ibrahim, E.L., Rewainy (2016). Evaluation of promising lines in rice (Oryza sativa L.) to agronomic and genetic performance under Egyptian conditions. Int. J. Agri. Agri. R. 8(3): 52-57.

Jennings, P.R., Coffman, W.F. and Kauffman, H.E. (1979).Rice improvement. Int. Rice. Res. Inst Los Banos, Manila, Phillipines.

Johnsson, H.W.; Robinson, H.F. and Comstock, R.E. (1955).Estimates of phenotypic and genotypic correlations in soybeans and their implications in selection. Agron. J. 47: 477-482.

Kumar, Mukul; Prakash, Nitendra and Sharma, Ph. Ranjit.(2009).Effectiveness of $\mathrm{F}_{2}$ and $\mathrm{F}_{3}$ plant selection for yield and its components in rice, Oryza, 46(1): 17-20.

Lingaiah, N. (2015).Genetic variability, heritability and genetic advance in rice (Oryza sativa L.). Asian Journal of Environmental Science, 10 (1): 110112.

Lokaprakash, R.; Shivashankar, G.; Shankare Gowda, B. T.; Mahadevappa, M. and Kulkarni, R. S. (1992). Study on genetic variability, heritability and Genetic advance in rice. Indian J. Genet., 52(4): 416-421.

Mohan, Lal and Chauhan, D.K. (2011).Studies of genetic variability, heritability and genetic advance in relation to yield traits in rice. Agricultural Science Digest. 31(3): 220222.

Panse, V. G. and Sukhatme, P. V. (1967).Genetics and qualitative 
characters in relation to plant breeding.

Indian J. Genet. 17: 312-328

Praveen, Pandey and Anurag, P.J. (2010).Estimation of genetic parameters in indigenous rice. $A A B$ Bioflux. 2(1): 79-8.

Rajendar, Reddy, M., Surendar. Raju. Ch, Dayakar. Reddy, T. and Narender Reddy, S. (2013). Study on genetic parameters in $\mathrm{F}_{2}$ generation involving aromatic rice genotypes $O$. sativa. Applied Biology and Pharmaceutical Technology, 4(4): 238-241.

Sanghera, Gulzar, S., Kashyap, C. Subhash (2012). Genetic Parameters and Selection Indices in $\mathrm{F}_{3}$ Progenies of Hill Rice. Not SciBiol, 4(4):110-114.

Shebeski, L.H. (1967).Wheat Breeding Proceedings of the Canadian Centennial Wheat Symposium modern Press, Saskatchewan, Canada, pp. 253.

Singh, Chandra, Mohan, Babu, G., Suresh, Kumar, Binod and Mehandi Suhel (2013). Analysis of quantitative variation and selection criteria for yield improvement in exotic germplasm of upland rice (Oryza sativa L.). The Bioscan 8(2): 485-492.
Sivasubramanian, P. and Menon, P. M. (1973).Genotypic and phenotypic variability in rice. Madras Agric.J.60: 1093-1096.

Tuhina, Khatun, M, Hanafi, M.M, Rafii, Yusop, M, Wong, M.Y, Salleh, F.M., Ferdous, J. (2015).Genetic variation, heritability, and diversity analysis of upland rice (Oryza sativa L.) genotypes based on quantitative traits. Biomed Res Int.: 290861

Verma S. K. and Mani S. C. (2000).Selection method and response to selection in rice. Indian J. Genet., 60: 477-481.

Vir, Om and Singh, B. B. (2005). Early generation testing and response to selection in three crosses of rice (Oryza sativa L.). Indian J. Genet. 65(2): 9698.

Whan B. R., Knight R. and Rathjan A. J. (1982).Response to selection for grain yield and harvest index in $F_{2}, F_{3}$ and $F_{4}$ derived lines of two wheat crosses Euphytica, 31: 139-150

Whan B.R., Rathjen A.J. and Night R. (1981). The relation between wheat lines derived from $F_{1}, \quad F_{3}, \quad F_{4}$ and $F_{5}$ generations for grain yield and harvest index. Euphytica30: 419-430.

\section{How to cite this article:}

Choudhary, A.K., Z.A. Haider, K. Prasad, S.B. Mishra, M. Chakraborty and Niraj Kumar. 2018. Selection Response and Genetic Variability for Yield and Its Component Traits of Rice (Oryza sativa L.). Int.J.Curr.Microbiol.App.Sci. 7(04): 931-945.

doi: https://doi.org/10.20546/ijcmas.2018.704.099 